\title{
Efficient Robust Reconstruction of Dynamic PET Activity Maps with Radioisotope Decay Constraints
}

\author{
Fei Gao ${ }^{1}$, Huafeng $\mathrm{Liu}^{2}$, and Pengcheng Shi ${ }^{1}$ \\ ${ }^{1}$ Golisano College of Computing and Information Sciences, \\ Rochester Institute of Technology, Rochester, NY, 14623, USA \\ 2 State Key Laboratory of Modern Optical Instrumentation, Zhejiang University, \\ Hangzhou, 310027, China
}

\begin{abstract}
Dynamic PET imaging performs sequence of data acquisition in order to provide visualization and quantification of physiological changes in specific tissues and organs. The reconstruction of activity maps is generally the first step in dynamic PET. State space $H_{\infty}$ approaches have been proved to be a robust method for PET image reconstruction where, however, temporal constraints are not considered during the reconstruction process. In addition, the state space strategies for PET image reconstruction have been computationally prohibitive for practical usage because of the need for matrix inversion. In this paper, we present a minimax formulation of the dynamic PET imaging problem where a radioisotope decay model is employed as physics-based temporal constraints on the photon counts. Furthermore, a robust steady state $H_{\infty}$ filter is developed to significantly improve the computational efficiency with minimal loss of accuracy. Experiments are conducted on Monte Carlo simulated image sequences for quantitative analysis and validation.
\end{abstract}

\section{Introduction}

Dynamic positron emission tomography (PET) is a molecular imaging technique that is used to monitor the spatiotemporal distribution of a radiotracer in vivo 1. Dynamic PET performs sequence of contiguous short-interval scans, and the reconstructed activity maps reflect the real-time concentration of radiotracers in tissues. The activity maps are conventionally reconstructed frame-by-frame from the dynamic data sequence with analytical or statistical methods, without taking into consideration potential temporal relations between frames. Further, through application of a priori models, parameter maps can be recovered to illustrate the dynamic changes of radiotracer kinetics. Together, the activity maps and the parameter maps provide visualization and quantification of physiological changes that indicate the functional states of specific tissues that are of significant research and clinical values [2]3.

Many research efforts have concentrated on the design of iterative statistical reconstruction algorithms for PET activity reconstruction [4, such as those based

T. Jiang et al. (Eds.): MICCAI 2010, Part III, LNCS 6363, pp. 571 578, 2010.

(C) Springer-Verlag Berlin Heidelberg 2010 
on expectation maximization(EM) and maximum a priori (MAP) criteria. These strategies assume the statistical distribution of acquired data to be known, which is not necessarily valid for corrected data from most clinical scanners. Liu et al. have first introduced a robust state space approach for PET image reconstruction [5], where they have utilized the robust $H_{\infty}$ filtering without any assumptions on the system and data noise statistics. However, as all other frame-by-frame methods, it ignores temporal constraints in the dynamic reconstruction of the image sequence. Recently, Gao et al. have extended the work to dynamic dualtracer PET reconstruction [6] where they introduced the temporal radioisotope decay model. However, the radio decay model is not incorporated as temporal constraints but rather used to separate two radioisotopes with drastically different half lives. Furthermore, a big shortcoming of these state space $H_{\infty}$ reconstruction algorithms is their computational complexity because of the need for large-scale matrix inversion, which has hindered their practical value.

In this paper, we concentrate on the development of an efficient and robust minimax activity reconstruction framework with radioisotope physics-based temporal constraints. The radio decay model, considering the natural decay property of the radioisotope, is introduced into the objective function as the temporal guidance for multi-frame image sequence reconstruction. The optimization problem is solved by continuous steady state $H_{\infty}$ filter for all image frames [7], which yields robust and accurate results with significant computation reduction compared to conventional $H_{\infty}$ filter reconstruction. Experiments are conducted on Monte Carlo simulated image sequences for quantitative analysis and validation.

\section{Theory}

\subsection{Radioisotope Decay Constrained Dynamic PET Imaging}

The projection equation of dynamic PET imaging can be formulated through an affine transform between the projection data and emission object as:

$$
y(t)=D x(t)+r(t)+s(t)
$$

where the emission sinogram data is represented by a vector $y$, and the activity of emission object is represented by $x . D$ is system probability matrix, which gives the probability of a photon emitted from $i^{t h}$ voxel being detected in projection $j^{t h}$ bin. $t$ is the time frame. $r$ and $s$ are the contributions of random coincidence events and scatter coincidence events. After the conventional online delayedwindow random correction, the equation(1) can be rewritten as:

$$
y(t)=D x(t)+e(t)
$$

here $e$ is an error vector, which represents unknown measurement uncertainties including scatter coincidence events.

In the conventional state space reconstruction of PET imaging, the distribution of the radioisotopes in the body is assumed to be temporally stationary corresponding to the autoradiographic model, however, in the real situation, the radioisotope will decay with time, and its activity at time $t$ should be 


$$
x=X_{0} e^{\frac{\ln (0.5)}{T} t}
$$

here $X_{0}$ is the initial activity distribution, and $T$ is the half life of the radioisotope. So the real-time change of radioisotope can be represented as

$$
\frac{d x}{d t}=X_{0} \frac{\ln (0.5)}{T} e^{\frac{\ln (0.5)}{T} t}
$$

then the dynamic change of radioisotope from one frame to the next can be obtained from the integral of equation(4). A general representation of state transition will be

$$
x(t+1)=H(t) x(t)+v(t)
$$

where $x(t)$ is the radioactivity concentration at time frame $t$, and $H(t)$ is a coefficient matrix for state transition at time frame $t . v(t)$ represents the uncertainties during state transition. With the introduction of decay model shown as equation(5), we are able to make use of the radioisotope's own temporal properties as constraints to guide our reconstruction.

\subsection{Robust Reconstruction Criterion}

Seeking solutions under uncertainties is a difficult problem. Robust discrete optimization seeks to identify solutions that will perform well under any circumstances. Although many criteria are available, one reasonable choice is the minimax criterion, which allows one to identify a robust solution as one that has the best worstcase performance. In general, a robust discrete optimization problem can be formulated as follows. Let $X$ be the set of all solutions, $E$ be the set of uncertainties of measurement in single time frame, $M$ be the set of uncertainties for state transition among time frames, and performance of a solution $x \in X$ under uncertainties $e \in E$ and $v \in M$ be $F(x, e, v)$, now the problem is to find the solution that has the best worst-case performance, which is the same as minimizing (over all solutions) the maximum (over all uncertainties) performance:

$$
\min _{x \in X} \max _{e \in E, v \in M} F(x, e, v)
$$

from the description of equation(2) and (5), the estimation of activity distribution $x(t)$ at time $t$ is not only computed based on measurement $y(t)$, but also affected by previous estimations, so we define a linear combination of $x(t)$ as

$$
z(t)=\mathcal{F} x(t)=g(x(k), H(k), v(k)) \quad k=1,2 \ldots t
$$

so the measurement of performance $F(x, e, v)$ is given by

$$
J=\frac{\sum\|z(t)-\hat{z}(t)\|_{Q(t)}^{2}}{\|x(0)-\hat{x}(0)\|_{p_{o}^{-1}}^{2}+\sum\left(\|v(t)\|_{V(t)^{-1}}^{2}+\|e(t)\|_{N(t)^{-1}}^{2}\right)}
$$

where the notation $\|x\|_{G}^{2}$ is defined as the square of the weighted (by $G$ ) $L_{2}$ norm of $x$ (i.e. $\left.\|x\|_{G}^{2}=x^{T} G x\right)$. $N(t), V(t)$ and $Q(t)$ are the weighting matrices for the 
measurement uncertainties, state transition uncertainties, and the estimation error at time $t$ respectively. $\hat{x}(0)$ is the initial estimate of the state. $p_{o}$ is the weighting matrix for the initial conditions. With the state estimate $\hat{x}(n)$ at time $n$, the measurement uncertainty $e(n)$ and state transition uncertainty $v(n)$ can be expressed as $e(n)=Y(n)-D \hat{x}(n)$ and $v(n)=\hat{x}(n+1)-H(n) \hat{x}(n)$. Then both the measurement-system matrix constraint and decay model constraint are implied in $J$. The optimal estimate $z(t)$ among all possible $\hat{z}(t)$ should satisfy:

$$
\|J\|_{\infty}=\sup J<\gamma^{2}
$$

where $\gamma^{2}>0$ is a prescribed level of disturbances. It is assumed that the $L_{2}$ norms of $e(t)$ and $v(t)$ exist. Then the minimax performance criterion of equation(8) where the estimator strategy $z(t)$ playing against the exogenous inputs $e(t), v(t)$ and the initial state $x(0)$ becomes

$$
\begin{array}{r}
\min _{z(t)-\hat{z}(t)} \max _{v, e, x(0)} J=\sum\|z(t)-\hat{z}(t)\|_{Q(t)}^{2}-\gamma^{2}\|x(0)-\hat{x}(0)\|_{p_{o}^{-1}}^{2} \\
-\gamma^{2} \sum\left(\|v(t)\|_{V(t)^{-1}}^{2}+\|e(t)\|_{N(t)^{-1}}^{2}\right)
\end{array}
$$

Now the problem becomes to solve the above objective function, and the decay model is successfully incorporated. Robust $H_{\infty}$ filter solution has already been presented in [5], so here we concentrate on a more efficient steady state $H_{\infty}$ filter.

\subsection{Efficient Implementation}

A matrix inverse is required at every time step in conventional $H_{\infty}$ filter in order to calculate the $H_{\infty}$ gain. Generally, inversion of small matrices is fairly easy, but the inversion of a large matrix will require more computational costs in a practical implementation. The steady state $H_{\infty}$ filter is much easier to be implemented in a system in which real-time computational effort or code size is a serious consideration [7].

The minimax objective function is given as equation(10), where the parameters $(N, V$ and $Q)$ are symmetric positive definite matrices based on the specific problem. Since the designed parameters of the underlying system can be treated as fixed values for input, then the steady state solution to the minimax problem can be obtained. Referring to $H_{\infty}$ filter, the steady state solution will be

$$
\begin{aligned}
K & =P S D^{T} N^{-1} \\
P & =H P S H^{T}+V \\
\hat{x}(m+1) & =H \hat{x}(m)+H K(m)(y(m)-D \hat{x}(m)) \\
S & =\left(I-\gamma^{-2} \bar{Q} P+D^{T} N^{-1} D P\right)^{-1} \\
\bar{Q} & =\mathcal{F}^{T} Q \mathcal{F}
\end{aligned}
$$

In order to have a solution to the problem, the following condition must be hold:

$$
P^{-1}-\gamma^{-2} \bar{Q}+D^{T} N^{-1} D>0
$$


If $\gamma^{-2}, \mathcal{F}, N$ or $Q$ is too large, or $D$ is too small, the $H_{\infty}$ estimator will have no solution. After the conditions above satisfied, equation(12) can be written as

$$
P=H\left[P^{-1}-\gamma^{-2} \bar{Q}+D^{T} N^{-1} D\right]^{-1} H^{T}+V
$$

Applying the matrix inversion lemma to the inverse of the above expression, we can get

$$
\begin{aligned}
P & =H P-P\left[P+\left(-\gamma^{-2} \bar{Q}+D^{T} N^{-1} D\right)^{-1}\right]^{-1} P H^{T}+V \\
& =H P H^{T}-H P\left[P+\left(-\gamma^{-2} \bar{Q}+D^{T} N^{-1} D\right)^{-1}\right]^{-1} P H^{T}+V
\end{aligned}
$$

Equation(16) is a discrete-time algebraic Riccati equation that can be solved by control system software or numerically iterating equation(12) until it converges to a steady state value.

The disadvantage of the steady state $H_{\infty}$ filter is that theoretically it does not perform as well as the time-varying filter. However, the reduced performance that is seen in the steady state $H_{\infty}$ solution is often a small fraction of the optimal performance, whereas the computational saving can be significant 7 .

\section{$3 \quad$ Experiments and Results}

The data set used for validation in this study was acquired by Monte Carlo simulations. Monte Carlo simulations can provide a relatively accurate reference for the development and assessment of new image reconstruction algorithms. Simulations in our study are performed using toolbox GATE. The simulated PET scanner is the newly designed full 3D whole body PET scanner Hamamatsu SHR74000 from Hamamatsu Photonics K.K.. The phantom chosen here for simulations is Zubal thorax phantom.

2 experiments were performed here. In the 1st experiment, the number of coincidence events acquired in every time frame was controlled to be similar, and this is in accordance with the conditions of conventional state space $H_{\infty}$ filter reconstruction. However, in real situation, it is not easy to control the number of coincidence events in every time frame, so we designed the 2nd experiment that sampled the data with the same time interval more intuitively. These 2 experiments are named as Experiment 1 and Experiment 2 in the following parts respectively. The initial activity per voxel was set to be low to avoid the contamination of random coincidence events. In Experiment 1, 20 time frames were acquired in all, and the total number of coincidence events was around 68k for every time frame; in Experiment 2, same 20 time frames were acquired in all, but the acquisition time was set to be 360 s for every time frame. The 2 experiments correspond to 2 different protocols for dynamic PET acquisition.

The simulation outputs were stored in sinograms. Before reconstruction, random correction, normalization correction, attenuation correction and scatter correction were performed properly. The sinograms were reconstructed as images of size $128 \times 128$ by $H_{\infty}$ filter without decay model, $H_{\infty}$ filter with decay model and steady state $H_{\infty}$ filter respectively. 


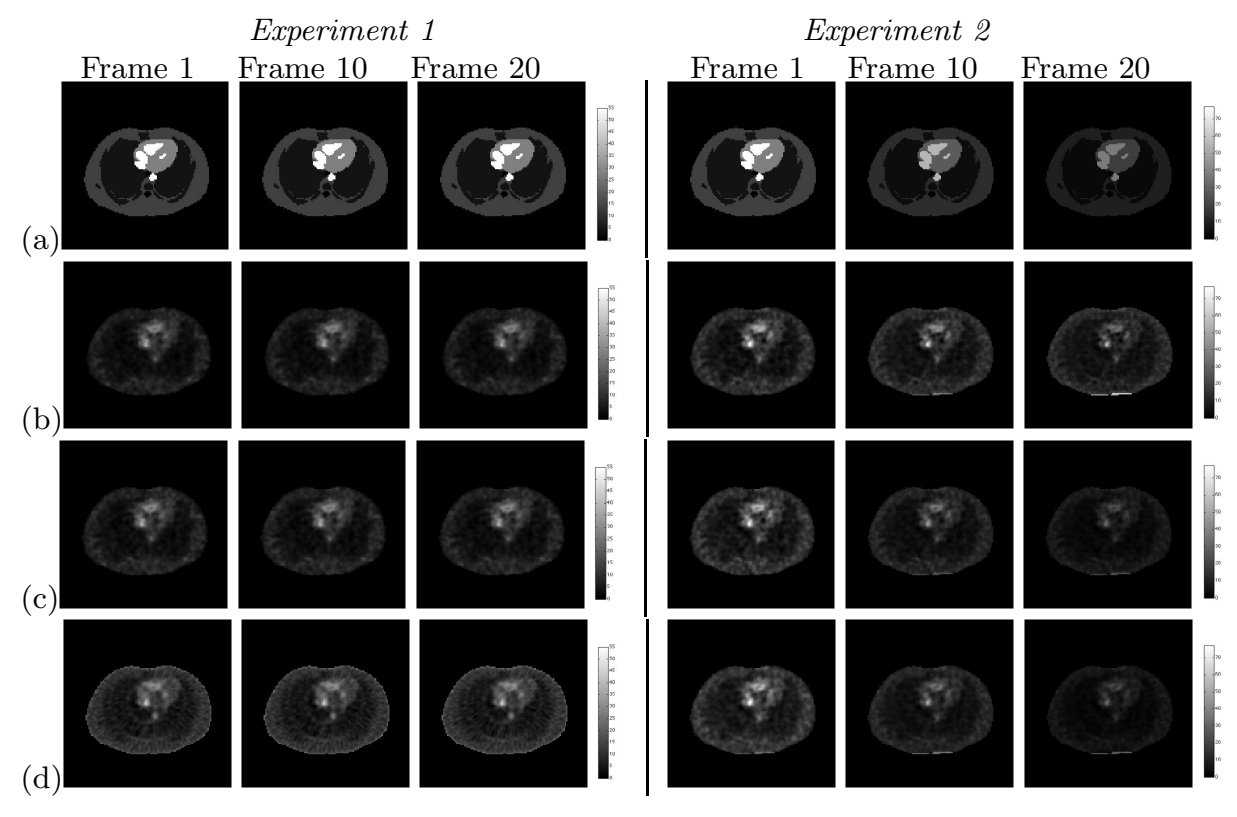

Fig. 1. True activity distributions and reconstructed images at time frame \#1, \#10 and $\# 20$ in 2 experiments. (a): true activity. (b): $H_{\infty}$ filter reconstruction without decay model. (c): $H_{\infty}$ filter reconstruction with decay model. (d): steady state $H_{\infty}$ filter reconstruction.

Fig. 1 gives the true activity distributions and reconstructed images of the central slice by 3 algorithms of both experiments at time frame \#1, \#10 and \#20. The reconstruction results from $H_{\infty}$ filter with decay model show better recovery of activity distributions than $H_{\infty}$ filter without decay model. Steady state $H_{\infty}$ filter can also reconstruct the activity distributions properly: the regions with high activity concentration are recovered well, but the non-activity regions are little overestimated.

Since these experiments are based on Monte Carlo simulations, we can get the true activity distributions at anytime exactly. In order to analyze the reconstruction results quantitatively, we define the Variance (Var) as follow: Var = $\frac{1}{n-1} \sum_{i=1}^{n} \frac{\left(x_{i}-\hat{x}_{i}\right)^{2}}{\hat{x}_{i}^{2}}$, where $x_{i}$ is the estimated activity value of pixel $i, \hat{x}_{i}$ is the true activity value of pixel $i$, and $n$ is the number of pixels.

The calculated variances between reconstruction results and true activity distributions through all time frames are plotted in Fig 2, and some results are extracted and listed in Table 1 for better understanding. From the quantitative analysis, in Experiment 1, due to the similar number of coincidence events acquired through all time frames, which corresponds to the assumptions of conventional $H_{\infty}$ filter reconstruction without decay model, reasonable results can be obtained through all the time frames by it; but in Experiment 2, the experiment conditions do not agree with the assumptions, so the calculated variances 


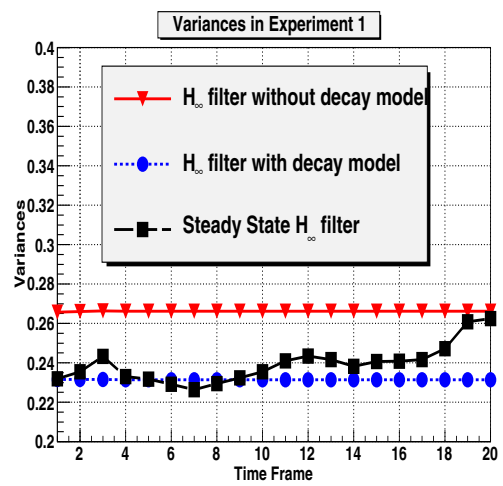

Experiment 1

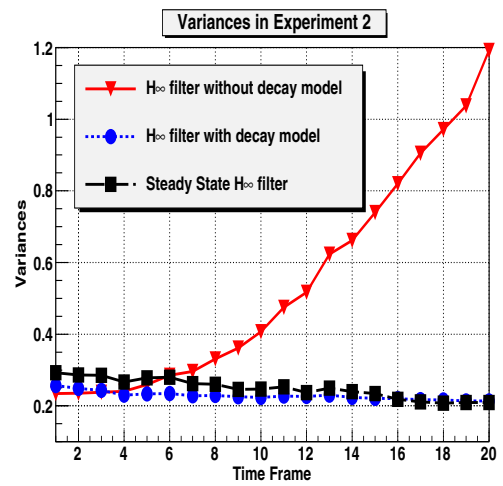

Experiment 2

Fig. 2. Variances of reconstructed images by 3 algorithms through all time frames

Table 1. Variances of reconstructed images by 3 algorithms in Experiment 1 and Experiment 2 at time frame \#1, \#5, \#10, \#15 and \#20

\begin{tabular}{|c|c|c|c|c|c|}
\hline Variances of Experiment 1 & Frame 1 & Frame 5 & Frame 10 & Frame 15 & Frame 20 \\
\hline$H_{\infty}$ without decay model & 0.2657 & 0.2664 & 0.2662 & 0.2662 & 0.2662 \\
\hline$H_{\infty}$ with decay model & 0.2316 & 0.2314 & 0.2314 & 0.2314 & 0.2314 \\
\hline Steady State $H_{\infty}$ & 0.2320 & 0.2318 & 0.2355 & 0.2408 & 0.2624 \\
\hline Variances of Experiment 2 & Frame 1 & Frame 5 & Frame 10 & Frame 15 & Frame 20 \\
\hline$H_{\infty}$ without decay model & 0.2342 & 0.2595 & 0.4066 & 0.7393 & 1.1914 \\
\hline$H_{\infty}$ with decay model & 0.2567 & 0.2331 & 0.2239 & 0.2206 & 0.2136 \\
\hline Steady State $H_{\infty}$ & 0.2922 & 0.2784 & 0.2470 & 0.2345 & 0.2090 \\
\hline
\end{tabular}

become larger as time increases, which means the quantitative accuracy of reconstruction results becomes worse. However, for the reconstruction results by $H_{\infty}$ filter with decay model, due to the introduction of the decay model, they are already more accurate than that reconstructed by $H_{\infty}$ filter without decay model in Experiment 1, furthermore, in Experiment 2, as a result of the temporal constraints of the decay model in reconstruction, the reconstruction results are much more stable through all the time frames, and the quantitative accuracy of reconstruction results are similar for all time frames and much better than that reconstructed by $H_{\infty}$ filter without decay model. The variances are reduced by $11 \%$ and $57 \%$ in average for Experiment 1 and Experiment 2 respectively. For steady state $H_{\infty}$ filter reconstruction, the results are not so stable as $H_{\infty}$ filter with decay model and the quantitative accuracies are little worse, but the reconstruction results are still much better than that reconstructed by $H_{\infty}$ filter without decay model, most importantly, significant computation saving is achieved by steady state $H_{\infty}$ filter shown as follow. 
Table 2. Time consumptions of 3 algorithms in Experiment 1 and Experiment 2

\begin{tabular}{|c|c|c|c|}
\hline Algorithm & $H_{\infty}$ without decay model & $H_{\infty}$ with decay model & Steady State $H_{\infty}$ \\
\hline Experiment 1 & $5536 \mathrm{~s}$ & $6228 \mathrm{~s}$ & $127 \mathrm{~s}$ \\
\hline Experiment 2 & $30531 \mathrm{~s}$ & $39924 \mathrm{~s}$ & $540 \mathrm{~s}$ \\
\hline
\end{tabular}

In this paper, all reconstructions are finished using MATLAB on a single 2.26GHz CPU, 12G RAM PC. The time consumptions are summarized in Table 2, Compared with $H_{\infty}$ filter, the computation costs were reduced by $99.7 \%$ in Experiment 1 and $98.6 \%$ in Experiment 2 by using steady state $H_{\infty}$ filter. This makes steady state $H_{\infty}$ filter more efficient for practical usage, furthermore, there are cases that steady state $H_{\infty}$ filter is used in embedded system, so further acceleration may be achieved by hardware. Related researches are underway.

\section{Conclusion}

An efficient and robust minimax activity reconstruction for dynamic PET imaging with radioisotope decay constraints is proposed in this paper. A physical decay model is introduced as temporal guidance in reconstruction. A robust steady state $H_{\infty}$ filter is adopted to solve the problem which leads to significant computation reduction and makes the algorithm more efficient and practical. Reconstruction results from simulation experiments show the robustness and improvement in quantitative accuracy of the minimax reconstruction with decay model. The time consumption analysis shows the efficiency of the steady state $H_{\infty}$ filter.

Acknowledgements. This work is supported in part by the National Basic Research Program of China (No: 2010CB732500).

\section{References}

1. Bailey, D.L., Townsend, D.W., Valk, P.E., Maisey, M.N.: Positron emission tomography: basic sciences. Springer, Heidelberg (2005)

2. Wernick, M., Infusino, E., Milosevic, M.: Fast spatio-temporal image reconstruction for dynamic PET. IEEE Transactions on Medical Imaging 18(3), 185-195 (1999)

3. Krestyannikov, E., Tohka, J., Ruotsalainen, U.: Joint penalized-likelihood reconstruction of time-activity curves and regions-of-interest from projection data in brain pet. Physics in Medicine and Biology 53(11), 2877-2896 (2008)

4. Qi, J., Leahy, R.: Iterative reconstruction techniques in emission computed tomography. Physics in Medicine and Biology 51, R54-R78 (2006)

5. Liu, H., Tian, Y., Shi, P.: PET image reconstruction: a robust state space approach. In: Christensen, G.E., Sonka, M. (eds.) IPMI 2005. LNCS, vol. 3565, pp. 197-209. Springer, Heidelberg (2005)

6. Gao, F., Liu, H., Jian, Y., Shi, P.: Dynamic dual-tracer pet reconstruction. In: Prince, J.L., Pham, D.L., Myers, K.J. (eds.) IPMI 2009. LNCS, vol. 5636, pp. 38 49. Springer, Heidelberg (2009)

7. Simon, D.: Optimal state estimation: kalman, $\mathrm{H}_{\infty}$ and nonlinear approaches. John Wiley and Sons, Chichester (2006) 